\title{
Análisis de las variables técnico-tácticas relacionas con el último golpe del punto en el tenis de alto rendimiento
}

Analysis of Technical-Tactical Variables Related to the Last Stroke of the Point in High Performance Tennis

\author{
Ángel Iván Fernández-García* (i) \\ Departamento de Fisiatría y Enfermería. Facultad de Ciencias de la Salud y del Deporte. Universidad de Zaragoza, Huesca, España \\ José María Juan-Campos \\ Facultad de Humanidades y Ciencias de la Educación, Universidad de Jaén, Jaén, España. \\ José María Giménez-Egido (D) \\ Facultad de Ciencias del Deporte. Campus Regional de Excelencia Internacional "Campus Mare Nostrum", Universidad de Murcia. Sports Perfor- \\ mance Analysis Association. España.
}

\section{Resumen}

El objetivo del presente trabajo fue analizar mediante diferentes variables técnico-tácticas el último golpe del punto en jugadores de tenis profesionales, así como estudiar las diferencias que puedan existir en dicha acción entre los ganadores y perdedores del partido y de los juegos. Fueron analizados el 100\% de los puntos $(n=1639)$ de la Copa de Maestros ATP 2014 disputada en pista rápida cubierta. Las variables técnico-tácticas analizadas fueron la zona de la pista desde la que se impactó la pelota, la acción técnica llevada a cabo, la zona en la que bota la pelota y el resultado final de la acción, mientras que las variables de resultado que se tuvieron en cuenta fueron ser el ganador o perdedor del partido o del juego. Los resultados muestran que los ganadores cometen menos errores y consiguen más golpes ganadores que los perdedores. Además, utilizan más el golpe de derecha y los golpes de red. Por su parte, los perdedores golpean con más frecuencia que los ganadores en el fondo de pista, mientras que estos últimos lo hacen más que sus rivales en la zona de red. Por tanto, los ganadores son más precisos y seguros, y utilizan tácticas más ofensivas que sus oponentes.

Palabras clave: deportes de raqueta, indicadores de rendimiento, análisis de la competición, golpe ganador, error.

\begin{abstract}
The aim of the present study was to analyze through different technical-tactical variables the last stroke of the point in professional tennis players, as well as to analyze the differences that may exist in this specific action between the winners and losers of the match and games. The sample analyzed were the $100 \%$ of the points $(n=1639)$ of the ATP Masters Cup 2014 played in indoor hard court. The variables analyzed were the last technical-tactical action, hitting area, ball landing location and stroke effectiveness, while the performance variables that considered were being the winner or loser of the match or the game. The results show that winners commit fewer errors and get more winners than the losers. In addition, they use more the forehand and net shots. Further, losers hit more frequently than the winners at the background while the latter do it more than their opponents in the net zone. Therefore, winners are more accurate and consistent, and use more offensive tactics than losers.
\end{abstract}

Keywords: racquet sports, performance indicators, match analysis, winner, error.

* Autor de correspondencia: Ángel Iván Fernández-García, angelivanfg@unizar.es

Recibido: 14-Mayo-2020

Aceptado: 14-Junio-2020

Publicado: Julio 2020

Como citar (APA): Fernández-García, A. I., Juan-Campos, J. M., \& Giménez-Egido, J. M. (2020). Análisis de las variables técnicotácticas relacionas con el último golpe del punto en el tenis de alto rendimiento. JUMP, (2), 48-56. https://doi.org/10.17561/jump.n2.5

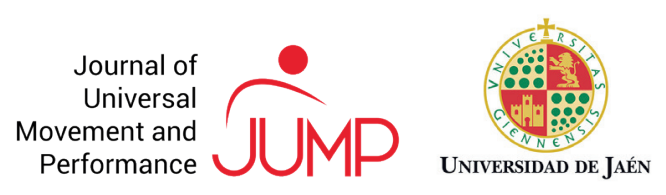




\section{Introducción}

El tenis es un deporte en el que los jugadores diseñan estrategias y toman decisiones técnicotácticas de manera constante para incrementar sus opciones de victoria basándose en sus propias fortalezas y debilidades, las de su rival y en factores ambientales o contextuales como la superficie de juego (Cui et al., 2017; Stare et al., 2015; Varas Caro \& Gómez-Ruano, 2016). La capacidad para analizar e interpretar lo que ocurre durante la competición ayuda a los jugadores a mejorar su rendimiento (Hughes \& Bartlett, 2002; Kovacs, 2007; O'Donoghue, 2013; Reid et al., 2016) y a los entrenadores a optimizar los procesos de entrenamiento, ya que las características de los mismos deben asemejarse a lo que ocurre durante la situación real de juego (Sánchez-Pay et al., 2015).

Debido a ello, el análisis de la competición en el tenis ha experimentado un auge en el ámbito de la investigación (Cui et al., 2018; Fitzpatrick et al., 2019; Klaus et al., 2017), siendo las áreas físico-fisiológica y la técnico-táctica las que han suscitado un mayor interés. Con respecto a la primera, son numerosos los trabajos que han tratado de describir las exigencias competitivas de este deporte en cuanto a las demandas fisiológicas (Fernandez-Fernandez et al., 2007), la duración de los puntos (Brown \& O'Donoghue, 2008), el número de golpeos por punto (Hornery et al., 2007; Johnson \& McHugh, 2006), los ratios de tiempo de trabajo y descanso (FernandezFernandez et al., 2008) o los desplazamientos de los jugadores durante un partido (MartínezGallego, Guzmán Luján, et al., 2013). Los estudios referentes al segundo grupo están principalmente orientados a obtener información sobre los indicadores de rendimiento (Cui et al., 2018), que son aquellas variables que determinan en mayor medida el resultado de un partido. Para ello, han centrado principalmente su atención en analizar las estadísticas de competición (Barbaros Tudor et al., 2014; Filipcic et al., 2015), las posiciones de los jugadores en la pista (Martínez-Gallego, et al., 2015; Martínez-Gallego, Guzmán Luján, et al., 2013; Martínez-Gallego, Guzmán, et al., 2013), - las variables que se asocian a la eficacia de los golpeos, como la velocidad o la colocación (Hizan et al., 2015; Reid et al., 2016; Whiteside \& Reid, 2017). Además, algunos trabajos han ido un paso más allá analizando patrones de rendimiento específicos en función de variables de tipo contextual, como la experiencia (Cui et al., 2017), el ranking (Cui et al., 2019), nivel de juego (Hizan et al., 2011; Stare et al., 2015), sexo de los jugadores (Hizan et al., 2015), ronda del torneo, o superficie de juego (Cui et al., 2019).

Para obtener información válida y fiable, tanto entrenadores como investigadores utilizan los numerosos avances tecnológicos y los métodos empleados en Ciencias del Deporte (Figueira et al., 2018), como los sistemas de seguimiento de la pelota ("ojo de halcón") (Reid et al., 2016; Whiteside \& Reid, 2017), los sensores inteligentes que se colocan en las raquetas (Giménez-egido et al., 2020) o el procesamiento de bancos de datos y técnicas de modelado estadístico (Cui et al., 2018). Sin embargo, a pesar del desarrollo de sistemas de registro automáticos, la metodología observacional sigue siendo uno de los métodos más utilizados para el análisis del juego (Teresa et al., 2011) especialmente en el estudio de parámetros tácticos (Hughes \& Bartlett, 2002). Sin embargo, a pesar de que se trata de una metodología común en investigación (Gillet \& Leroy, 2009; Hizan et al., 2015; P. O'Donoghue \& Ingram, 2001), no existe un consenso claro a la hora de emplearla en este deporte. En este sentido, algunos trabajos han intentado establecer unos criterios específicos de análisis, como la herramienta desarrollada por Gorospe Egaña et al. (2005) para el tenis de individuales o la de Hizan et al., (2010) para el estudio de las zonas de bote en las acciones de servicio y resto. Sin embargo, y desde nuestro conocimiento, la publicada recientemente Torres-Luque et al. (2018) es una de las más completas para analizar el tenis en su modalidad de individuales por el amplio abanico de variables que contempla.

En base a los resultados obtenidos por estudios previos, no hay duda de que el tenis está evolucionando hacia un estilo más ofensivo en el que los jugadores/as intentan presionar a sus rivales desde el inicio del punto (Filipčič et al., 2008; Katić et al., 2011; Stare et al., 2015), siendo las acciones de servicio y resto las que han recibido una mayor atención en el campo de la investigación por su mayor influencia en el juego (Gillet \& Leroy, 2009; Grambow et al., 2020; Hizan et al., 2011; Krause et al., 2019). Sin embargo, a pesar de que el comienzo de los puntos condiciona el desarrollo del juego y las posibilidades de ganar o perder los puntos (O'Donoghue \& Brown, 2008), son escasos 
los estudios que han focalizado su atención en comprobar cómo finalizan los mismos, o dicho en otras palabras, analizar las características del último golpe del punto en el que tienen lugar los golpes ganadores y los errores y comprobar qué diferencias existen cuando son ejecutados por los ganadores o perdedores.

Por ello, el objetivo del presente trabajo fue analizar mediante metodología observacional el último golpeo del punto teniendo en cuenta la zona de la pista desde la que se ejecute, la acción técnica llevada a cabo, la zona en la que bota la pelota y el resultado final de la acción, así como estudiar las diferencias que puedan existir en dicha acción entre los ganadores y perdedores del partido y de los juegos en partidos de tenis profesional de alto rendimiento.

\section{Material y método}

\section{Muestra}

La muestra estuvo compuesta por el 100\% de los puntos disputados ( $n=1639)$ durante los 15 partidos de la Copa de Maestros ATP disputada en Londres en pista cubierta en el año 2014. El torneo reúne a final de temporada a los 8 jugadores con mejor ranking mundial de la ATP (Association Tennis Profesionals). El formato del torneo es diferente al resto de competiciones oficiales de la temporada, ya que se compone de una primera fase de liga en la que se forman 2 grupos de 4 jugadores, y una segunda fase eliminatoria a la que acceden los 2 primeros clasificados de cada grupo para disputar las semifinales y final. Todos los competidores eran diestros. Para el análisis, sólo se tuvieron en cuenta los últimos golpeos de cada punto, siempre y cuando estos no se ejecutaran con la acción del servicio, por lo que los servicios directos y dobles faltas no se incluyeron en el estudio. Además, aquellos partidos que finalizaran con abandono o descalificación de alguno de los dos jugadores ( $n=1$ ) fueron excluidos del estudio tal y como procedieron trabajos previos (O'Donoghue \& Ingram, 2001), por lo que la muestra final fue de 1436 golpes.

\section{Procedimiento}

El estudio se llevó a cabo en 4 fases. En la primera, se realizó la grabación de todos los partidos a través de la señal emitida por el canal
Teledeporte mediante un disco duro multimedia Woxter i-Cube 3900 con capacidad para 4 Therabays. Durante la segunda se llevó a cabo el entrenamiento de los 4 observadores que participaron en el estudio, todos ellos Graduados en Educación Primaria (Mención Educación Física) o en Ciencias de la Actividad Física y del Deporte, con experiencia en el entrenamiento de tenis. Para ello se confeccionó un "Manual de Instrucciones" en el que se definieron las categorías y variables objetivo de estudio (Tabla 1) en base al instrumento diseñado y validado para tal fin por Torres-Luque et al. (2018). Dicho entrenamiento se llevó a cabo siguiendo las recomendaciones de Villaseñor et al. (2001). La calidad del dato se comprobó a través de los coeficientes de Tau de Kendall, Pearson y Spearman y los niveles aptos de fiabilidad intra e inter observador (Anguera, 2003) mediante el coeficiente Kappa de Cohen (Cohen, 1968). Durante la tercera fase se llevó a cabo el visionado de los partidos mediante el programa de análisis de vídeo Kinovea 8.15 para Windows, utilizándose la herramienta "perspective grid" para delimitar las áreas de bote y golpeo (Giménez-Egido et al., 2020). Así mismo se registró el dato de manera simultánea en una hoja de observación diseñada para tal fin con el software Microsoft Excel versión 2010 para Windows. En la cuarta y última fase se realizó el análisis estadístico.

\section{Análisis estadístico}

Se empleó el programa estadístico SPSS 20.0 para Windows, llevándose a cabo un análisis descriptivo de frecuencias para cada una de las categorías objeto de estudio utilizando como variables de agrupación el resultado final de los jugadores en partido y en el juego perteneciente al golpe analizado.

\section{Resultados}

La Tabla 2 muestra la frecuencia con la que los jugadores ejecutan último golpe del punto, siendo los perdedores los que lo hacen en un porcentaje más alto de ocasiones. Además, los datos señalan que el porcentaje de errores es superior al de golpes ganadores, acrecentándose dicha diferencia cuando son los perdedores los que llevan a cabo la última acción del punto. Por contra, los ganadores consiguen un mayor porcentaje de golpes ganadores. 
Tabla 1. Categorías y variables analizadas en el último golpe del punto

\begin{tabular}{|c|c|c|}
\hline Categoría & Variables & Observaciones \\
\hline \multirow{2}{*}{$\begin{array}{l}\text { Jugador que ejecuta el último golpe } \\
\text { según el resultado final del partido }\end{array}$} & Ganador del partido & Vencedor del partido \\
\hline & Perdedor del partido & Perdedor del partido \\
\hline \multirow{2}{*}{$\begin{array}{l}\text { Jugador que ejecuta el último golpe } \\
\text { según el resultado final del juego }\end{array}$} & Ganador del juego & $\begin{array}{l}\text { Jugador que termina ganando el juego en el que se disputa el punto } \\
\text { analizado }\end{array}$ \\
\hline & Perdedor del juego & $\begin{array}{l}\text { Jugador que termina perdiendo el juego en el que se disputa el punto } \\
\text { analizado }\end{array}$ \\
\hline \multirow{5}{*}{ Acción técnica } & Derecha & \\
\hline & Revés & \\
\hline & Volea de derecha & \\
\hline & Volea de revés & \\
\hline & Remate & \\
\hline \multirow{4}{*}{$\begin{array}{l}\text { Zona de golpeo según una división } \\
\text { de la pista en profundidad* }\end{array}$} & Fondo de pista & $\begin{array}{l}\text { Área que se extiende desde } 1 \mathrm{~m} \text { por detrás de la línea de fondo hasta el } \\
\text { final de la pista }\end{array}$ \\
\hline & Cerca de la línea & $\begin{array}{l}\text { Área que se extiende desde la línea de fondo hasta } 1 \mathrm{~m} \text { por detrás de } \\
\text { esta }\end{array}$ \\
\hline & Dentro de pista & Área comprendida entre la línea de fondo y la línea de saque \\
\hline & Zona de red & Área comprendida entre la línea de saque y la red \\
\hline \multirow{3}{*}{$\begin{array}{l}\text { Zona de golpeo según una división } \\
\text { de la pista lateral* }\end{array}$} & Zona central & $\begin{array}{l}\text { Área comprendida desde la red hasta el fondo de pista y separada por } \\
2,74 \mathrm{~m} \text { de las líneas laterales de individuales }\end{array}$ \\
\hline & Zona derecha & $\begin{array}{l}\text { Área comprendida entre la red y el fondo de pista que se extiende desde } \\
\text { el límite derecho de la zona central situado a 2,74 m de la línea lateral } \\
\text { derecha de individuales y el límite lateral derecho de la pista }\end{array}$ \\
\hline & Zona izquierda & $\begin{array}{l}\text { Área comprendida entre la red y el fondo de pista que se extiende desde } \\
\text { el límite izquierdo de la zona central situado a } 2,74 \mathrm{~m} \text { de la línea lateral } \\
\text { izquierda de individuales y el límite lateral izquierdo de la pista }\end{array}$ \\
\hline \multirow{9}{*}{ Zona de bote** } & Corta centro & $\begin{array}{l}\text { Área comprendida entre la red y la línea de saque, quedando a una dis- } \\
\text { tancia de } 2,74 \text { m de las líneas laterales. }\end{array}$ \\
\hline & Corta derecha & $\begin{array}{l}\text { Área comprendida entre la red y la línea de saque, que se extiende 2,74 } \\
\text { m desde la línea lateral derecha de individuales hacia el centro de la } \\
\text { pista }\end{array}$ \\
\hline & Corta izquierda & $\begin{array}{l}\text { Área comprendida entre la red y la línea de saque, que se extiende 2,74 } \\
\text { m desde la línea lateral izquierda de individuales hacia el centro de la } \\
\text { pista }\end{array}$ \\
\hline & Media centro & $\begin{array}{l}\text { Área comprendida entre } 2,74 \mathrm{~m} \text { por delante de la línea de fondo hasta } \\
\text { la línea de saque y queda separada por } 2,74 \mathrm{~m} \text { de las líneas laterales de } \\
\text { individuales }\end{array}$ \\
\hline & Media derecha & $\begin{array}{l}\text { Área comprendida desde } 2,74 \mathrm{~m} \text { por delante de la línea de fondo hasta } \\
\text { la línea de saque y se extiende } 2,74 \mathrm{~m} \text { desde la línea lateral derecha de } \\
\text { individuales hacia el centro de la pista }\end{array}$ \\
\hline & Media izquierda & $\begin{array}{l}\text { Área comprendida desde } 2,74 \mathrm{~m} \text { por delante de la línea de fondo hasta } \\
\text { la línea de saque y se extiende } 2,74 \mathrm{~m} \text { desde la línea lateral izquierda de } \\
\text { individuales hacia el centro de la pista }\end{array}$ \\
\hline & Profunda centro & $\begin{array}{l}\text { Área que se extiende } 2,74 \text { m desde la línea de fondo hacia la línea de } \\
\text { saque y queda separada 2,74 m de las líneas laterales }\end{array}$ \\
\hline & Profunda derecha & $\begin{array}{l}\text { Área que se extiende } 2,74 \text { m desde la línea de fondo hacia la línea de } \\
\text { saque y se prolonga 2,74 desde la línea lateral derecha de individuales } \\
\text { hacia el centro de la pista }\end{array}$ \\
\hline & Profunda izquierda & $\begin{array}{l}\text { Área que se extiende } 2,74 \text { m desde la línea de fondo hacia la línea de } \\
\text { saque y se prolonga } 2,74 \text { desde la línea lateral izquierda de individuales } \\
\text { hacia el centro de la pista }\end{array}$ \\
\hline \multirow{2}{*}{ Eficacia } & Error & $\begin{array}{l}\text { Cualquier golpeo que no entre dentro de los límites reglamentarios de la } \\
\text { pista o no supere la red }\end{array}$ \\
\hline & Golpe ganador & $\begin{array}{l}\text { Cualquier golpeo que entre dentro de los límites reglamentarios y no } \\
\text { pueda ser interceptado por el oponente }\end{array}$ \\
\hline
\end{tabular}


Por otro lado, la Tabla 3 muestra la frecuencia con la que se ejecutan las diferentes acciones técnicas en el último golpe del punto, siendo los golpes de fondo (derecha y revés) las más utilizadas independientemente del resultado obtenido en el partido y en el juego. Por su parte, los golpes de red (volea de derecha, volea de revés y remate) son menos frecuentes, especialmente cuando son los perdedores los responsables de golpear en última instancia.

En referencia a la zona de la pista en la que se lleva a cabo el último golpe del punto, la Tabla 4 muestra los resultados cuando la pista queda dividida mediante 3 cortes paralelos a la red, los cuales forman 4 zonas que van desde el fondo de pista hasta la red. En ella se pone de manifiesto que los últimos impactos de cada punto se ejecutan principalmente por detrás de la línea de fondo y mayormente cerca de esta. Además, se observa una tendencia en los ganadores a finalizar más puntos en la red que los perdedores.

Por su parte, la Tabla 5 refleja la localización del impacto cuando la pista queda fragmentada por dos líneas perpendiculares a la red dividen la pista en 3 zonas, una central y dos laterales. Los resultados ponen de manifiesto que cuando son los ganadores los que ejecutan el último golpe, suelen hacerlo en más ocasiones en la zona central de lo que lo hacen los perdedores, que tienden a golpear más que estos en las zonas laterales. Además, también se aprecia que, independientemente del resultado, la zona de la pista con una mayor acumulación de impactos en el último golpe es la izquierda, en la que habitualmente se realizan los golpeos de revés o derecha escorada.

Por último, en la Tabla 6 se aprecian las zonas de bote de la pelota cuando en el último golpe del punto los jugadores consiguen un golpe ganador. Independientemente del resultado obtenido en el partido y en el juego, los jugadores obtienen una mayor cantidad de golpes ganadores cuando dirigen su último golpe sobre la zona media derecha del oponente, siendo la zona profunda derecha la segunda en eficacia.

Tabla 2. Frecuencia de ejecución y eficacia del último golpe del punto según el resultado del partido y el juego

\begin{tabular}{lcccc}
\hline & Ganador del partido & Perdedor del partido & Ganador del juego & Perdedor del juego \\
\hline Total & $44,5 \%(n=639)$ & $55,5 \%(n=797)$ & $38,4 \%(n=552)$ & $61,6 \%(n=884)$ \\
Error* & $31,5 \%(n=453)$ & $44,9 \%(n=645)$ & $21,9 \%(n=315)$ & $54,5 \%(n=783)$ \\
Golpe ganador* & $13,0 \%(n=186)$ & $10,6 \%(n=152)$ & $16,5 \%(n=237)$ & $7,0 \%(n=101)$ \\
Error** & $70,9 \%(n=453)$ & $80,9 \%(n=645)$ & $57,1 \%(n=315)$ & $88,6 \%(n=783)$ \\
Golpe ganador** & $29,1 \%(n=186)$ & $19,1 \%(n=152)$ & $42,9 \%(n=237)$ & $11,4 \%(n=101)$ \\
\hline
\end{tabular}

Tabla 3. Frecuencia de uso de las acciones técnicas ejecutadas en el último golpe del punto teniendo en cuenta el resultado del partido y del juego

\begin{tabular}{|c|c|c|c|c|}
\hline & Ganador del partido & Perdedor del partido & Ganador del juego & Perdedor del juego \\
\hline Derecha & $46,8 \%(n=299)$ & $47,8 \%(n=381)$ & $48,7 \%(n=269)$ & $46,5 \%(n=411)$ \\
\hline Revés & $42,1 \%(n=269)$ & $43,3 \%(n=345)$ & $34,4 \%(n=190)$ & $48,0 \%(n=424)$ \\
\hline Volea de derecha & $3,0 \%(n=19)$ & $2,4 \%(n=19)$ & $4,9 \%(n=27)$ & $1,2 \%(n=11)$ \\
\hline Volea de revés & $3,4 \%(n=22)$ & $2,8 \%(n=22)$ & $4,3 \%(n=24)$ & $2,3 \%(n=20)$ \\
\hline Remate & $4,7 \%(n=30)$ & $3,8 \%(n=30)$ & $7,6 \%(n=42)$ & $2,0 \%(n=18)$ \\
\hline Total & $100 \%(n=639)$ & $100 \%(n=797)$ & $100 \%(n=552)$ & $100 \%(n=884)$ \\
\hline
\end{tabular}

Tabla 4. Localización del impacto en el último golpe del punto cuando la pista queda dividida en 4 zonas que van desde la red al fondo de pista, teniendo en cuenta el resultado del partido y del juego

\begin{tabular}{|c|c|c|c|c|}
\hline & Ganador del partido & Perdedor del partido & Ganador del juego & Perdedor del juego \\
\hline Fondo de pista & $22,8 \%(n=146)$ & $24,7 \%(n=197)$ & $19,4 \%(n=107)$ & $26,7 \%(n=236)$ \\
\hline Cerca de la línea & $45,7 \%(n=292)$ & $43,3 \%(n=345)$ & $36,8 \%(n=203)$ & $49,1 \%(n=434)$ \\
\hline Dentro de pista & $19,7 \%(n=126)$ & $23,0 \%(n=183)$ & $25,9 \%(n=143)$ & $18,8 \%(n=166)$ \\
\hline Zona de red & $11,7 \%(n=75)$ & $9,0 \%(n=72)$ & $17,9 \%(n=99)$ & $5,4 \%(n=48)$ \\
\hline Total & $100 \%(n=639)$ & $100 \%(n=797)$ & $100 \%(n=552)$ & $100 \%(n=884)$ \\
\hline
\end{tabular}


Tabla 5. Localización del impacto en el último golpe del punto cuando la pista queda dividida en 3 zonas, una central y dos laterales, teniendo en cuenta el resultado del partido y del juego

\begin{tabular}{lcccc}
\hline & Ganador del partido & Perdedor del partido & Ganador del juego & Perdedor del juego \\
\hline Zona central & $22,4 \%(n=143)$ & $18,4 \%(n=147)$ & $30,4 \%(n=168)$ & $13,8 \%(n=122)$ \\
Zona derecha & $32,9 \%(n=210)$ & $34,0 \%(n=271)$ & $26,6 \%(n=147)$ & $37,8 \%(n=334)$ \\
Zona izquierda & $44,8 \%(n=286)$ & $47,6 \%(n=379)$ & $42,9 \%(n=237)$ & $48,4 \%(n=428)$ \\
\hline Total & $100 \%(n=639)$ & $100 \%(n=797)$ & $100 \%(n=552)$ & $100 \%(n=884)$ \\
\hline
\end{tabular}

Tabla 6. Zona en la que bota la pelota tras ejecutar un golpe ganador en el último golpe del punto teniendo en cuenta el resultado del partido y del juego

\begin{tabular}{|c|c|c|c|c|}
\hline & Ganador del partido & Perdedor del partido & Ganador del juego & Perdedor del juego \\
\hline Corta centro & $3,2 \%(n=6)$ & $1,3 \%(n=2)$ & $3,0 \%(n=7)$ & $1,0 \%(n=1)$ \\
\hline Corta derecha & $10,2 \%(n=19)$ & $10,7 \%(n=16)$ & $11,1 \%(n=26)$ & $9,0 \%(n=9)$ \\
\hline Corta izquierda & $11,8 \%(n=22)$ & $10,7 \%(n=16)$ & $13,2 \%(n=31)$ & $7,0 \%(n=7)$ \\
\hline Media centro & $0,5 \%(n=1)$ & $0,0 \%(n=0)$ & $0,4 \%(n=1)$ & $0,0 \%(n=0)$ \\
\hline Media derecha & $21,5 \%(n=40)$ & $26,2 \%(n=39)$ & $20,0 \%(n=47)$ & $32,0 \%(n=32)$ \\
\hline Media izquierda & $16,7 \%(n=31)$ & $14,8 \%(n=22)$ & $18,3 \%(n=43)$ & $10,0 \%(n=10)$ \\
\hline Profunda centro & $0,0 \%(n=0)$ & $2,7 \%(n=4)$ & $0,9 \%(n=2)$ & $2,0 \%(n=2)$ \\
\hline Profunda derecha & $20,4 \%(n=38)$ & $18,1 \%(n=27)$ & $17,9 \%(n=42)$ & $23,0 \%(n=23)$ \\
\hline Profunda izquierda & $15,6 \%(n=29)$ & $15,4 \%(n=23)$ & $15,3 \%(n=36)$ & $16,0 \%(n=16)$ \\
\hline Total & $100 \%(n=186)$ & $100 \%(n=149)$ & $100 \%(n=235)$ & $100 \%(n=100)$ \\
\hline
\end{tabular}

\section{Discusión}

Este estudio tuvo como objetivo analizar mediante metodología observacional el último golpeo del punto teniendo en cuenta la zona de la pista desde la que se ejecute, la acción técnica llevada a cabo, la zona en la que bota la pelota y el resultado final de la acción, así como estudiar las diferencias que puedan existir en dicha acción entre los ganadores y perdedores del partido y de los juegos en partidos de tenis profesional de alto rendimiento. Los principales hallazgos del estudio ponen de manifiesto: 1) con el último golpeo del punto, se cometen más errores que golpes ganadores; 2) los perdedores ejecutan con mayor frecuencia el último golpeo del punto y además cometen una mayor proporción de errores y menos golpes ganadores; 3 ) independientemente del resultado conseguido en el partido y en el juego, las acciones técnicas más utilizadas en el último golpe del punto son la derecha y el revés, aunque se aprecia una tendencia a que los ganadores finalicen los puntos en más ocasiones que sus rivales con golpes de red, especialmente los ganadores de los juegos; 4) la zona en de la pista en las que se impacta con mayor frecuencia el último golpe del punto, es justo detrás de la línea de fondo y en el sector izquierdo o zona de revés, aunque los ganadores golpean en mayor medida que los perdedores en la zona central y también cerca de la red; 5) independientemente del resultado, las zonas de bote más eficaces para conseguir un golpe ganador son la media derecha, seguida por la profunda derecha.

A pesar de que el análisis de la competición está en auge en el tenis, pocos datos existen en la actualidad acerca de las variables técnicotácticas que influyen en el último golpe del punto. Nuestro estudio pone de manifiesto que la gran mayoría de los puntos finaliza con un error de alguno de los jugadores (76,4\%), aunque el porcentaje correspondiente a los cometidos por los perdedores es superior (44,9\%), que el de los ganadores (31,5\%). En este sentido, el estudio de Fitzpatrick et al. (2019) llegó a una conclusión similar, estableciendo en un 66,7\% el total de puntos que finalizan con error, siendo superior el porcentaje de errores cometidos por los perdedores del partido (36,7\%) que el de los ganadores (30,0\%). Sin embargo, a pesar de que existe una ligera diferencia entre ambos trabajos, ésta puede deberse a que, a diferencia del estudio 
citado previamente, el nuestro no tuvo en cuenta para el análisis aquellos puntos que finalizaban con un saque directo o una doble falta, por lo que presumiblemente los porcentajes se igualarían. Por su parte, el 23,6\% de los últimos impactos, son golpes ganadores. A diferencia de lo comentado en el párrafo anterior, en este caso son los ganadores de los partidos los que contribuyen con un mayor porcentaje (13,0\%) que los perdedores (10,6\%). Si a los porcentajes obtenidos por Fitzpatrick et al. (2019) para los golpes ganadores se les elimina la parte correspondiente a los saques directos, los resultados son prácticamente idénticos a los nuestros (13,0\% en los ganadores Vs $11,4 \%$ de los perdedores). Por tanto y en consenso con los datos obtenidos por otros estudios (Cui et al., 2017; Fernández-García et al., 2019; Martínez-Gallego et al., 2013, 2017), se pone de manifiesto que los ganadores son más eficaces en el último golpe del punto, ya que consiguen una mayor cantidad de golpes ganadores y cometen menos errores.

Con respecto a la acción técnica con la que se ejecuta el último golpe del punto, los resultados muestran una clara predominancia de los golpes de fondo (derecha y revés), sobre los de red (voleas y remate). En este sentido, se observa como los ganadores golpean con más frecuencia de derecha que de revés, siendo esta diferencia mayor en los ganadores del juego que en los del partido. Además, los ganadores tienden a utilizar más los golpes de red que los perdedores. Todo lo anterior, unido a que los jugadores que pierden golpean más veces desde el fondo de pista, y a que los que ganan ejecutan con más frecuencia el último golpe en zona de red, nos lleva a la conclusión que los ganadores utilizan estrategias más ofensivas. Estos datos son apoyados por otros estudios cuyos resultados muestran que los jugadores profesionales tienden a golpear más veces (ratio 1,24:1) (Reid et al., 2016) y a conseguir más golpes ganadores (FernándezGarcía et al., 2019) con la derecha que con el revés. Por ello, la derecha es considerada generalmente como el golpe de fondo más ofensivo y con el que los jugadores hacen más daño a sus rivales. Por otro lado, los estudios de Martínez-Gallego et al. (2013 y 2017) concluyen que los ganadores de los partidos pasan más tiempo en zonas ofensivas que los perdedores y que estos últimos recorren una mayor distancia y a más velocidad que los ganadores, lo que indica que habitualmente los ganadores adoptan tácticas más ofensivas y llevan a sus rivales a jugar en zonas más alejadas y en posiciones más forzadas, razón que probablemente está asociada al hecho de que éstos comentan más errores (Pollard et al., 2006) y finalicen menos puntos en la red. Con respecto a esto último, no sólo parece que los jugadores que pierden suben menos a la red, sino que además, cuando lo hacen obtienen una menor eficacia (67-71\% ganadores Vs 61-64\% perdedores), tal y como concluyen trabajos anteriormente mencionados (Fernández-García et al., 2019; Fitzpatrick et al., 2019).

Por último y analizando las zonas de bote de los golpes ganadores, se observa como las zonas de mayor efectividad son la media y profunda derecha, lo que indica que los golpes ganadores se producen sobre la zona del golpe de derecha del rival. Si a dicha información le añadimos los datos previos, puede intuirse una tendencia a nivel táctico-estratégico en la que los ganadores tratan de provocar el error jugando sobre el revés de sus oponentes o generar ventaja para posteriormente buscar el golpe ganador sobre la zona contraria con su golpe de derecha, bien con un ángulo cruzado desde la zona natural o con un golpe paralelo desde la zona izquierda (golpe de derecha escorada).

En esta línea de investigación, futuros estudios deberían profundizar en un análisis más exhaustivo de los golpes ganadores y los errores con el objetivo de conocer cuáles son los golpes, zonas de la pista y zonas de bote que permiten incrementar la posibilidad de conseguir golpes ganadores o reducir los errores. Además, sería interesante comprobar si los patrones de rendimiento del último golpe se modifican en función de variables contextuales como el género y nivel de los jugadores o la superficie de juego.

\section{Conclusión}

Existen diferencias en los patrones técnicotácticos del último golpe del punto en función del resultado en el partido y en el juego. Los resultados muestran que los ganadores cometen menos errores y consiguen más golpes ganadores que los perdedores. Además, utilizan más el golpe de derecha y los golpes de red. Por su parte, los perdedores golpean con más frecuencia que los ganadores en el fondo de pista, mientras que estos últimos lo hacen más que sus rivales en la zona de red. Por tanto, los ganadores son más precisos y seguros, y utilizan tácticas más ofensivas que sus oponentes. 


\section{Aplicación práctica}

El análisis de la competición permite a los jugadores incrementar su rendimiento competitivo ya que les da la posibilidad de obtener una información muy valiosa sobre los puntos fuertes y débiles propios y de los rivales. Además, ayuda a los entrenadores a conocer las demandas específicas de la competición y por tanto a mejorar la eficacia de los procesos de entrenamiento.

\section{Conflicto de intereses}

\section{Los autores declaran no tener ningún conflicto de intereses.}

\section{Referencias}

Anguera, M. T. (2003). Observational methods (general). Encyclopedia of Psychological, 2, 632-637.

Barbaros Tudor, P., Zečić, M., \& Matković, B. (2014). Differences Between 2010 and 2011 Performance Indicators of Tennis Play At the Grand Slam Tournaments. Kineziologija, 46(Supplement 1), 102-107.

Brown, E., \& O'Donoghue, P. (2008). Gender and Surface Effect on Elite Tennis Strategy. Coaching \& Sport Science Review, 46, 9-11.

Cohen, J. (1968). Weighted kappa: Nominal scale agreement provision for scaled disagreement or partial credit. Psychological Bulletin, 70(4), 213-220. https://doi.org/10.1037/h0026256

Cui, Y., Gómez, M. Á., Gonçalves, B., Liu, H., \& Sampaio, J. (2017). Effects of experience and relative quality in tennis match performance during four Grand Slams. International Journal of Performance Analysis in Sport, 17(5), 783-801. https://doi.org/10.1080/24748668.2017.1399325

Cui, Y., Gómez, M. Á., Gonçalves, B., \& Sampaio, J. (2018). Performance profiles of professional female tennis players in grand slams. PLOS ONE, 13(7), 1-18. https://doi.org/10.1371/journal.pone.0200591

Cui, Y., Liu, H., Liu, H., \& Gómez, M. Á. (2019). Data-driven analysis of point-by-point performance for male tennis player in Grand Slams. Motricidade, 15(1), 49-61. https://doi.org/10.6063/motricidade.16370

Fernandez-Fernandez, J., Mendez-Villanueva, A., FernandezGarcia, B., \& Terrados, N. (2007). Match activity and physiological responses during a junior female singles tennis tournament. British Journal of Sports Medicine, 41(11), $711-$ 716. https://doi.org/10.1136/bjsm.2007.036210

Fernandez-Fernandez, J., Sanz-Rivas, D., Fernandez-Garcia, B., \& Mendez-Villanueva, A. (2008). Match activity and physiological load during a clay-court tennis tournament in elite female players. Journal of Sports Sciences, 26(14), 1589-1595. https://doi.org/10.1080/02640410802287089

Fernández-García, Á. I., Blanca-Torres, J. C., Nikolaidis, P. T., \& TorresLuque, G. (2019). Differences in competition statistics between winners and losers in male and female tennis players in Olympic Games. German Journal of Exercise and Sport Research, 49(3), 313-318. https://doi.org/10.1007/s12662-019-00608-y

Figueira, B., Gonçalves, B., Folgado, H., Masiulis, N., CallejaGonzález, J., \& Sampaio, J. (2018). Accuracy of a basketball indoor tracking system based on standard bluetooth low energy channels (NBN23®). Sensors (Switzerland), 18(6). https://doi.org/10.3390/s18061940
Filipcic, A., Zecic, M., Reid, M., Crespo, M., Panjan, A., \& Nejc, S. (2015). Differences in performance indicators of elite tennis players in the period 1991- 2010. Journal of Physical Education and Sport, 15(4), 671-677. https://doi.org/10.7752/jpes.2015.04102

Filipčič, T., Filipčič, A., \& Berendijaš, T. (2008). Comparison of game characteristics of male and female tennis players at Roland Garros 2005. Sport Sciences, 38(3), 151-155. http://10.0.93.21/TSS.2017.24.4-

Fitzpatrick, A., Stone, J. A., Choppin, S., \& Kelley, J. (2019). A simple new method for identifying performance characteristics associated with success in elite tennis. International Journal of Sports Science and Coaching, 14(1), 43-50. https://doi.org/10.1177/1747954118809089

Gillet, E., \& Leroy, D. (2009). A notational analysis of elite tennis serve and serve-return strategies on slow surface. Journal of Strength, 23(2), 532-539.

Gimenez-Egido, J. M., Ortega-Toro, E., Palao, J. M., VerdúConesa, I., \& Torres-Luque, G. (2020). Effect of Modification Rules in Competition on Technical-Tactical Action in Young Tennis Players (Under-10). Frontiers in Psychology, 10, 1-15. https://doi.org/10.3389/fpsyg.2019.02789

Giménez-egido, J. M., Ortega, E., Verdu-conesa, I., Cejudo, A., \& Torres-luque, G. (2020). Using smart sensors to monitor physical activity and technical-tactical actions in junior tennis players. International Journal of Environmental Research and Public Health, 17(3), 12-15. https://doi.org/10.3390/ijerph17031068

Gorospe Egaña, G., Hernández-Mendo, A., Anguera, M. T., \& Martínez de Santos, R. (2005). Desarrollo y optimización de una herramienta observacional en el tenis de individuales. Psicothema, 17(1), 123-127.

Grambow, R., O'Shannessy, C., Born, P., Meffert, D., \& Vogt, T. (2020). Serve efficiency development at wimbledon between 2002 and 2015: A longitudinal approach to impact tomorrow's tennis practice. Human Movement, 21(1), 65-72. https://doi.org/10.5114/hm.2020.88155

Hizan, H., Whipp, P. R., \& Reid, M. (2010). Validation of Match Notation (A Coding System) in Tennis. Journal of Quantitative Analysis in Sports, 6(3). https://doi.org/10.2202/1559-0410.1223

Hizan, H., Whipp, P., \& Reid, M. (2011). Comparison of serve and serve return statistics of high performance male and female tennis players from different age-groups. International Journal of Performance Analysis in Sport, 17(2), 365-375. https://doi.org/10.1080/24748668.2011.11868556

Hizan, H., Whipp, P., \& Reid, M. (2015). Gender differences in the spatial distributions of the tennis serve. International Journal of Sports Science and Coaching, 10(1), 87-96. https://doi.org/10.1260/1747-9541.10.1.87

Hornery, D. J., Farrow, D., Mujika, I., \& Young, W. (2007). Fatigue in Tennis. Sports Medicine, 37(3), 199-212. https://doi.org/10.2165/00007256-200737030-00002

Hughes,M.D.,\&Bartlett,R.M.(2002). Theuseofperformanceindicators in performance analysis. Journal of Sports Sciences, 20(10), 739-754. https://doi.org/10.1080/026404102320675602

Johnson, C. D., \& McHugh, M. P. (2006). Performance demands of professional male tennis players. British Journal of Sports Medicine, 40(8), 696-699. https://doi.org/10.1136/bjsm.2005.021253

Katić, R., Milat, S., Zagorac, N., \& Durović, N. (2011). Impact of game elements on tennis match outcome in Wimbledon and Roland Garros 2009. Collegium Antropologicum, 35(2), 341-346. http://www.ncbi.nlm.nih.gov/pubmed/21755700

Klaus, A., Bradshaw, R., Young, W., O'Brien, B., \& Zois, J. (2017). Success in national level junior tennis: Tactical perspectives. International Journal of Sports Science and Coaching, 12(5), 618-622. https://doi.org/10.1177/1747954117727792

Kovacs, M. S. (2007). Tennis physiology: Training the competitive athlete. Sports Medicine, 37(3), 189-198. https://doi.org/10.2165/00007256-200737030-00001

Krause, L. M., Buszard, T., Reid, M., Pinder, R., \& Farrow, D. (2019). Assessment of elite junior tennis serve and return practice: A cross-sectional observation. Journal of Sports Sciences, 37(24), 2818-2825. https://doi.org/10.1080/02640414.2019.1665245 
Mart, R., Directores, G., Guzm, F., \& Vuckovic, G. (2015). Análisis técnico-táctico y de los desplazamientos de tenistas profesionales en pista rápida. 1-205

Martínez-Gallego, R., Guzmán, J. F., Crespo, M., Ramón-Llin, J. \& Vučković, G. (2017). Technical, tactical and movement analysis of men 's professional tennis on hard courts courts. The Journal of Sports Medicine and Physical Fitness, 59(1). https://doi.org/10.23736/s0022-4707.17.07916-6

Martínez-Gallego, R., Guzmán, J. F., James, N., Ramón-Llin, J., Crespo, M., \& Vuckovic, G. (2013). The relationship between the incidence of winners/errors and the time spent in different areas of the court in elite tennis. Journal of Human Sport and Exercise, 8(3), 2-5. https://doi.org/10.4100/jhse.2013.8.Proc3.05

Martínez-Gallego, R., Guzmán Luján, J. F., James, N., Pers, J., Ramón-Llin, J., \& Vuckovic, G. (2013). Movement characteristics of elite tennis players on hard courts with respect to the direction of ground strokes. Journal of Sports Science and Medicine, 12(2), 275-281

O'Donoghue, G. P., \& Brown, E. (2008). The Importance of Service in Grand Slam Singles Tennis. International Journal of Performance Analysis in Sport, 8(3), 70-78. https://doi.org/10.1080/24748668.2008.11868449

O'Donoghue, P., \& Ingram, B. (2001). A notational analysis of elite tennis strategy. Journal of Sports Sciences, 19(2), 107-115. https://doi.org/10.1080/026404101300036299

O'Donoghue, Peter. (2013). Rare events in tennis. International Journal of Performance Analysis in Sport, 13(2), 535-552. https://doi.org/10.1080/24748668.2013.11868668

Pollard, G., Cross, R., \& Meyer, D. (2006). An analysis of ten years of the four grand slam men's singles data for lack of independence of set outcomes. Journal of Sports Science and Medicine, 5(4), $561-566$.

Reid, M., Morgan, S., \& Whiteside, D. (2016). Matchplay characteristics of Grand Slam tennis: implications for training and conditioning. Journal of Sports Sciences, 34(19), $1791-$ 1798. https://doi.org/10.1080/02640414.2016.1139161

Sánchez-Pay, A., Palao, J. M., Torres-Luque, G., \& Sanz-Rivas, D. (2015). Differences in set statistics between wheelchair and conventional tennis on different types of surfaces and by gender. International Journal of Performance Analysis in Sport, 15(3) 1177-1188. https://doi.org/10.1080/24748668.2015.11868860

Stare, M., Žibrat, U., \& Filipčič, A. (2015). Stroke effectivness in professional and junior tennis = Učinkovitost udarcev $\mathrm{v}$ profesionalnem in mladinskem tenisu. Kinesiologia Slovenica, 27(2), 39-50

Teresa, M., Argilaga, A., Villaseñor, A. B., Hernández, A., Luis, J., \& López, L. (2011). Diseños observacionales: ajuste y aplicación en psicología del deporte. Cuadernos de Psicología Del Deporte, $77(2), 63-76$.

Torres-Luque, G., Fernández-García, Á. I., Cabello-Manrique, D. Giménez-Egido, J. M., \& Ortega-Toro, E. (2018). Design and validation of an observational instrument for the technicaltactical actions in singles tennis. Frontiers in Psychology, 9(DEC), 1-10. https://doi.org/10.3389/fpsyg.2018.02418

Varas Caro, I., \& Gómez-Ruano, Á. M. (2016). Análisis Notacional en Jugadores de Tenis de Élite En Función de las Variables Contextuales Notational Analysis of Elite Tennis Players in Contextual Variables. Kronos, 15(151), 1-12.

Villaseñor, A. B., Losada, J. I, \& Anguera, M. T. (2001). Diseños observacionales, cuestión clave en el proceso de la metodología observacional. Metodología de Las Ciencias Del Comportamiento, 3(2), 135-160. https://dialnet.unirioja.es/servlet/articulo?codigo=2809601

Whiteside, D., \& Reid, M. (2017). Spatial characteristics of professional tennis serves with implications for serving aces: A machine learning approach. Journal of Sports Sciences, 35(7), 648-654. https://doi.org/10.1080/02640414.2016.1183805 